\title{
Supporting Information \\ How Does the Step on Graphite Surface Impact Ice Nucleation?
}

\author{
Quanming $\mathrm{Xu}^{1}$, Hao $\mathrm{Wang}^{1}$, Jianyang $\mathrm{Wu}^{1}$, and Zhisen Zhang ${ }^{1,{ }^{*}}$ \\ 1 Department of Physics, Research Institute for Biomimetics and Soft Matter, Jiujiang Research Institute and Fujian Provincial Key \\ Laboratory for Soft Functional Materials Research, Xiamen University, Xiamen 361005, PR China \\ *Email: zhangzs@xmu.edu.cn
}

Table of Contents

Figure S1. Schematic diagram of step structure adopted in MD simulation with periodic boundary conditions. 2

Figure S2: Ice nucleation process on graphite at $218 \mathrm{~K}$ in this work 2

Figure S3: Ice nucleation process on graphite at $218 \mathrm{~K}$ in previous report........ 3

Figure S4. Snapshots of ice nucleation on surfaces with step structure of single graphite height in $\mathrm{V}_{1} \mathrm{~S}_{1}$ system. .3

Figure S5. Snapshots of ice nucleation and freezing efficiency of surfaces with step structure of various height. .4

Figure S6. Snapshots of ice nucleation of cooling ramps in $V_{1} S_{1}$ system. .4

Figure S7. Snapshots of ice nucleation of cooling ramps in $V_{1} S_{2}$ system. .5

Figure S8. Snapshots of ice nucleation of cooling ramps in $\mathrm{V}_{2} \mathrm{~S}_{2}$ system. .5

Figure S9. Snapshots of ice nucleation of isothermal simulations in $V_{1} S_{1}$ system. .6

Figure S10. Snapshots of ice nucleation of isothermal simulations in $V_{1} S_{2}$ system..........

Figure S11. Snapshots of ice nucleation of isothermal simulations in $\mathrm{V}_{2} \mathrm{~S}_{2}$ system.........8

Figure S12. MD simulation system with nanogrooves of graphite. .9

\footnotetext{
* Corresponding Emails: zhangzs@xmu.edu.cn
} 


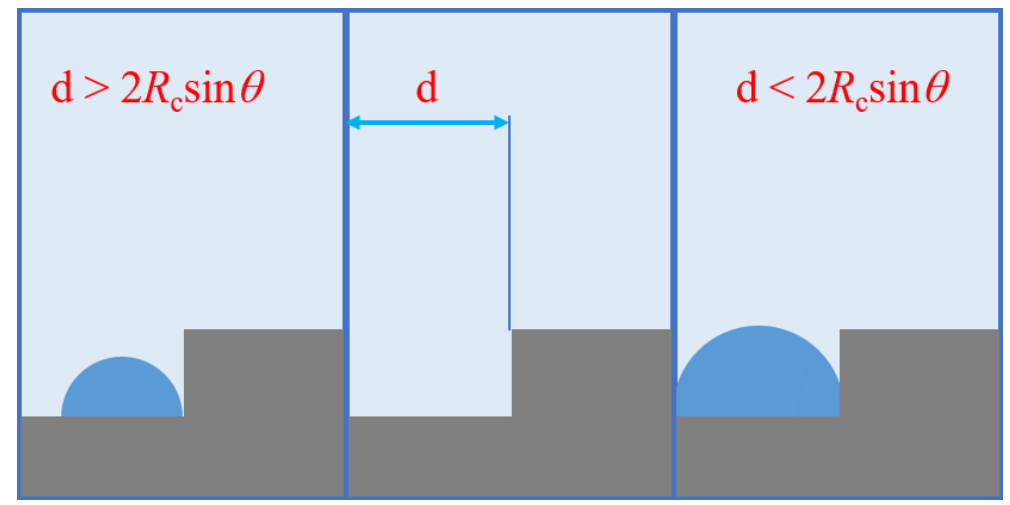

Figure S1. Schematic diagram of step structure adopted in MD simulation with periodic boundary conditions. For the $\mathrm{V}_{1} \mathrm{~S}_{1}$ system (with a surface area of $5 \times 5 \mathrm{~nm}^{2}$ ), a nanogroove with width $d$ of $2.5 \mathrm{~nm}$ is formed in systems with step structure due to the periodic boundary conditions. The ice nucleus with a critical size can only be impacted by one step edge of nanogrooves while $d>2^{R_{c}} \sin \theta$, where $R_{c}$ is the critical size of ice nucleus (about $1 \mathrm{~nm}$ at $205 \mathrm{~K}$ (Nature 2011, 479 (7374), 506-508)); $\theta$ is the contact angle of ice nucleus on graphite $\left(86.6^{\circ}\right.$ in the report (Phys. Rev. E 2015, 91 (5), 052402)). The substrates are colored by gray. The ice nucleus is colored by blue.

A
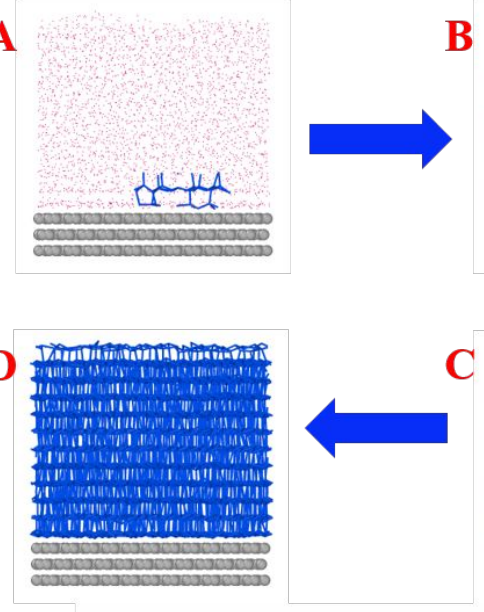

B

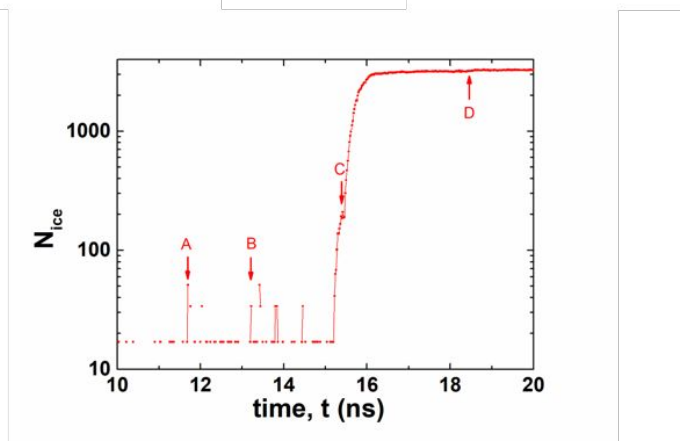

Figure S2: Ice nucleation process on graphite at $218 \mathrm{~K}$ in this work (ice clusters identified by OVITO). Panel A and B show subcritical ice nuclei. Panel $\mathrm{C}$ shows the successful nucleation event at the surface with the formation of the critical nucleus composed of $\sim 150$ water molecules. Panel D shows the fully crystallized water. The lower panel shows the size of ice nucleus (number of water molecules in the largest ice cluster, $N_{i c e}$ ) as a function of the simulation time, points A-B-C-D correspond to the snapshots of the upper panels. The points of subcritical were relatively sparse (Ice clusters were subcritical nuclei of less than 10 water molecules, and more of them 
were 0 ) because the time interval of the points of crystallization trajectories in our simulations was relatively large. Ice nucleation at about 15.2 ns and the critical nucleus contains about 110 water molecules.
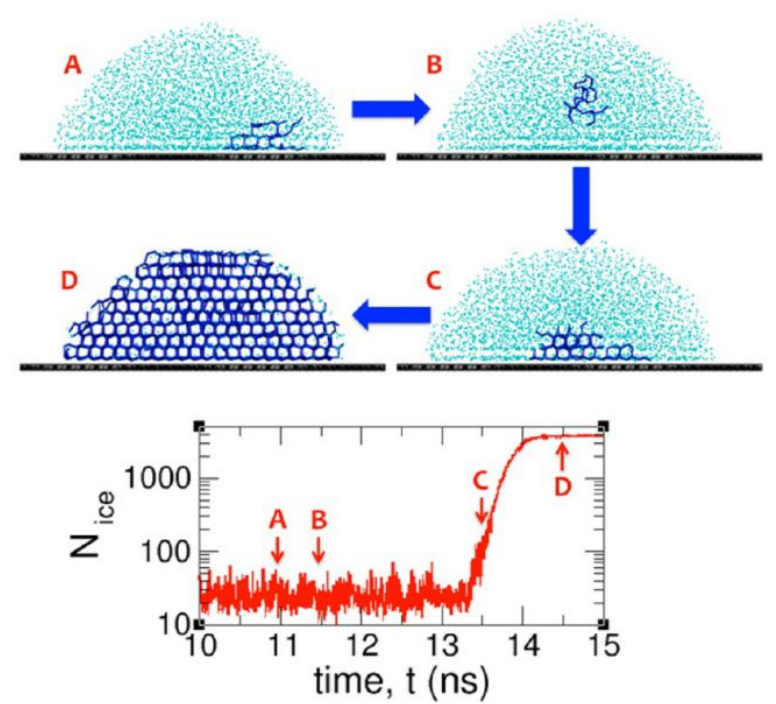

Figure S3: Ice nucleation process on graphite at $218 \mathrm{~K}$ in previous report (J. Am. Chem. Soc. 2014, 136 (8), 3156-3164). Panel A and B show subcritical ice nuclei. Panel $C$ shows the successful nucleation event at the surface with the formation of the critical nucleus composed of $\sim 150$ water molecules. Panel $D$ shows the fully crystallized water. The lower panel shows the size of ice nucleus (number of water molecules in the largest ice cluster, $N_{i c e}$ ) as a function of the simulation time, points A-B-C-D correspond to the snapshots of the upper panels. Ice nucleation at about $13.5 \mathrm{~ns}$ and the critical nucleus contains about 110 water molecules.
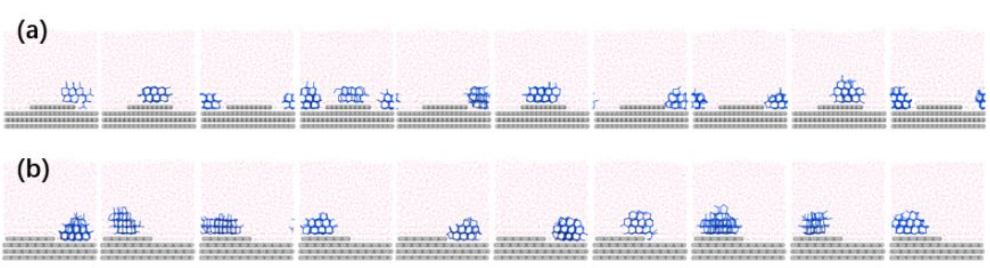

Figure S4. Snapshots of ice nucleation on surfaces with step structure of single graphite height in $V_{1} S_{1}$ system. There are armchair and zigzag structure for the step structure with single graphite height. Panel (a), (b) show armchair and zigzag structure respectively. Surfaces are shown with gray balls, liquid water with red dots and ice crystallites with blue sticks. Freezing efficiency of armchair and zigzag structure are $13.3 \pm 2.7 \mathrm{~K}$ and $13.8 \pm 2.6 \mathrm{~K}$. 


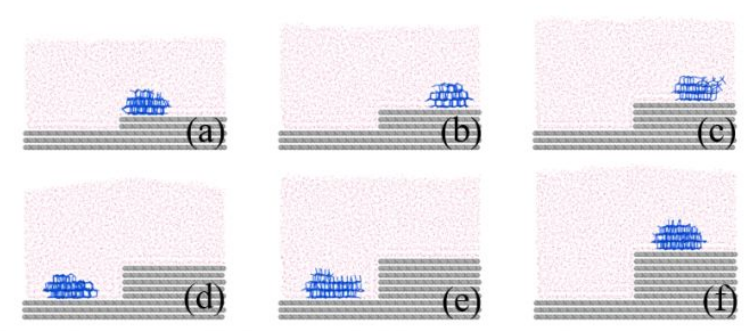

(g)

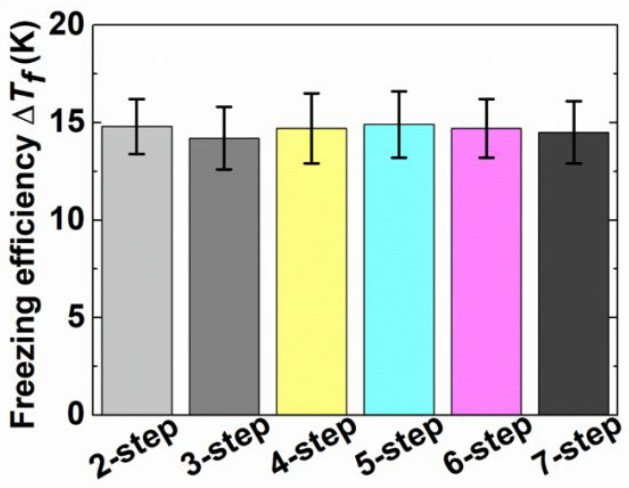

Figure S5. Snapshots of ice nucleation and freezing efficiency of surfaces with step structure of various height. (a) 2-step, (b) 3-step, (c) 4-step, (d) 5-step, (e) 6-step, (f) 7-step represent step structure with height of two-layer, three-layer, four-layer, five-layer, six-layer and seven-layer graphite. Surfaces are shown with gray balls, liquid water with red dots and ice crystallites with blue sticks. Panel (g) shows the freezing efficiency of surfaces.

(a)

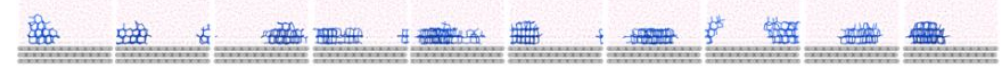

(b)

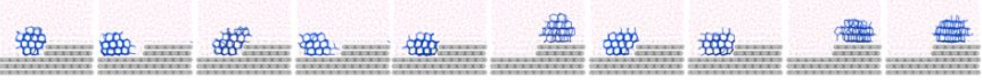

(c)

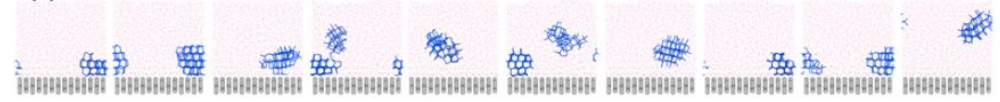

Figure S6. Snapshots of ice nucleation of cooling ramps in $\mathrm{V}_{1} \mathrm{~S}_{1}$ system. Panel (a), (b) and (c) represent flat surface, step structure and lateral surface, respectively. Surfaces are shown with gray balls, liquid water with red dots and ice crystallites with blue sticks. 
(a)

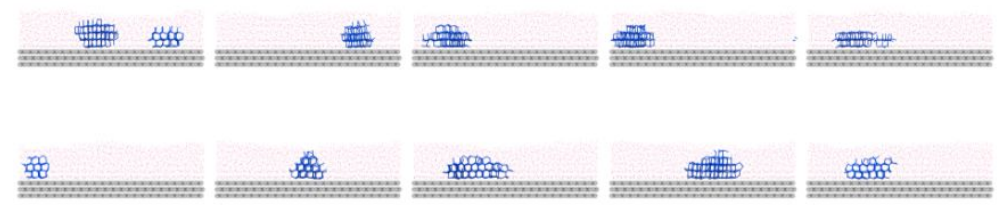

(b)

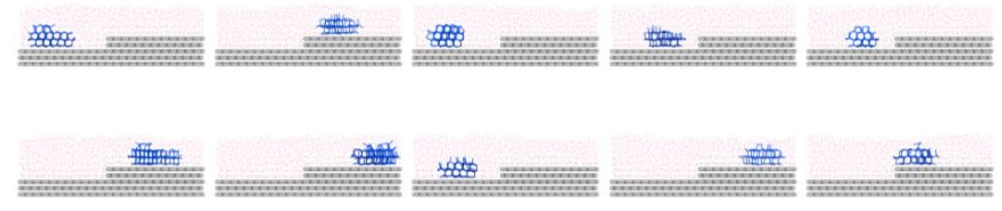

(c)

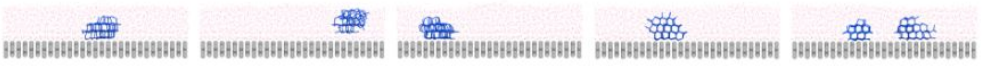

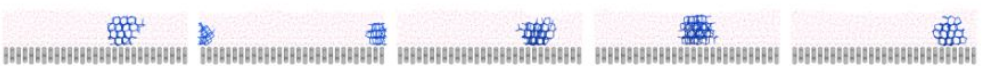

Figure S7. Snapshots of ice nucleation of cooling ramps in $\mathrm{V}_{1} \mathrm{~S}_{2}$ system. Panel (a), (b) and (c) represent flat surface, step structure and lateral surface respectively. Surfaces are shown with gray balls, liquid water with red dots and ice crystallites with blue sticks.

(a)

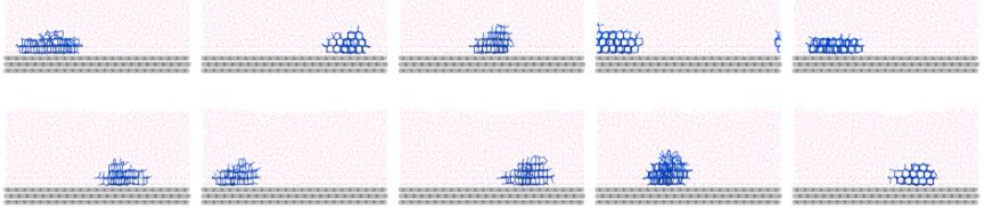

(b)

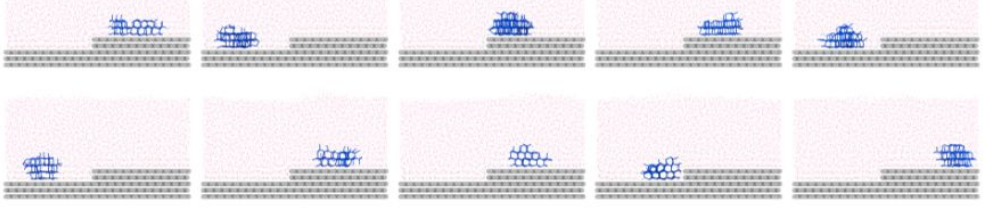

(c)

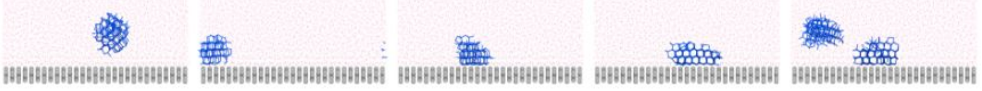

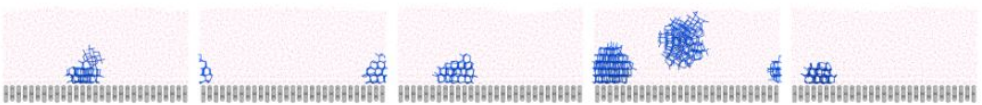

Figure S8. Snapshots of ice nucleation of cooling ramps in $\mathrm{V}_{2} \mathrm{~S}_{2}$ system. Panel (a), (b) and (c) represent flat surface, step structure and lateral surface respectively. Surfaces are shown with gray balls, liquid water with red dots and ice crystallites with blue sticks. 
(a)

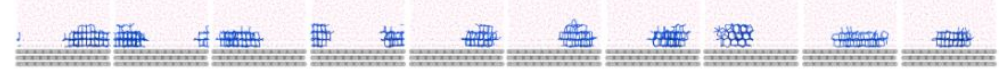

蛙

(b)

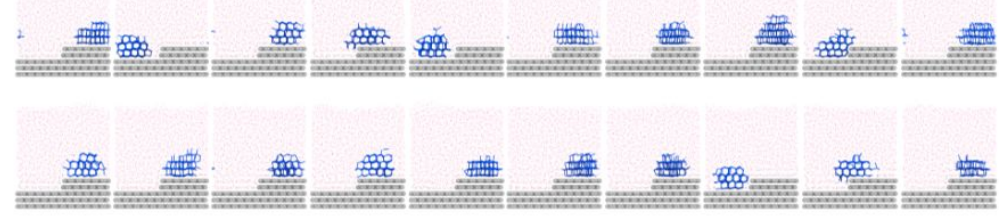

(c)

3.

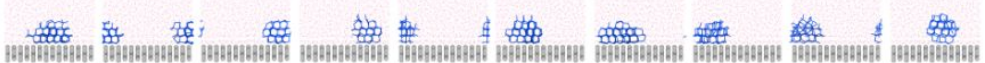

Figure S9. Snapshots of ice nucleation of isothermal simulations in $V_{1} S_{1}$ system. Panel (a), (b) and (c) represent flat surface, step structure and lateral surface respectively. Surfaces are shown with gray balls, liquid water with red dots and ice crystallites with blue sticks. 
(a)

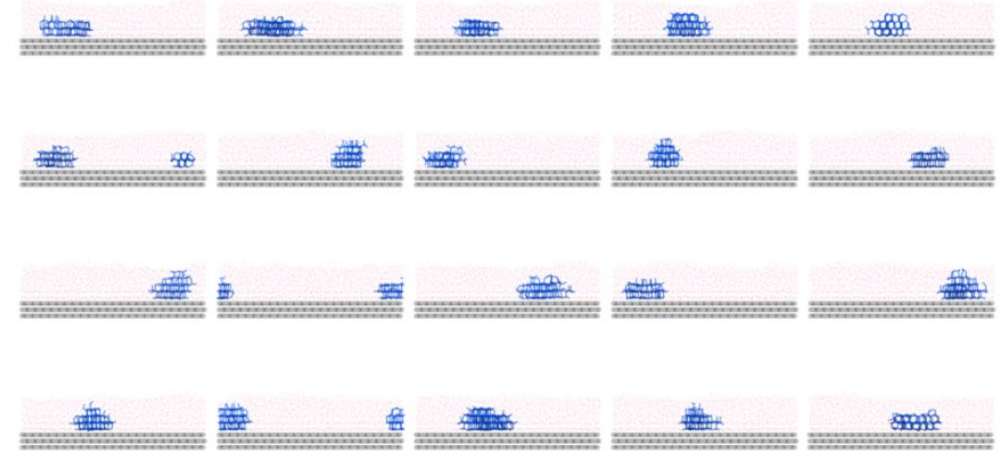

(b)
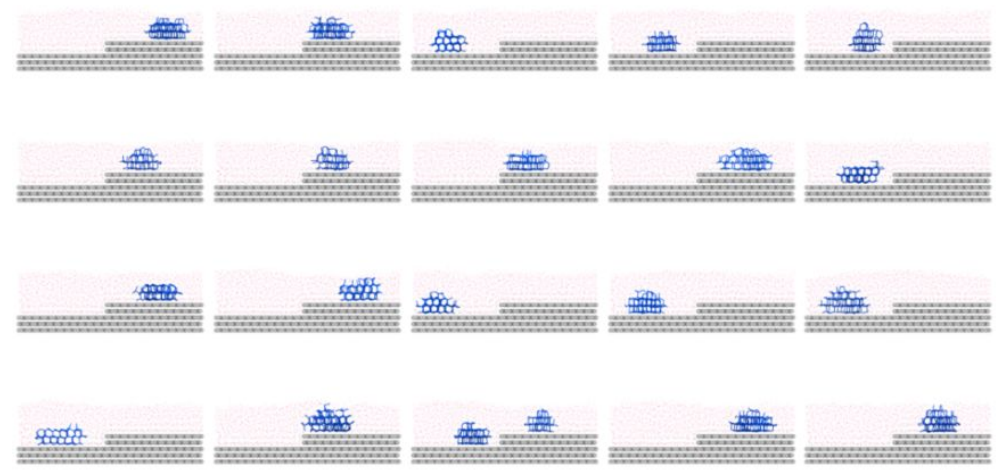

(c)

息

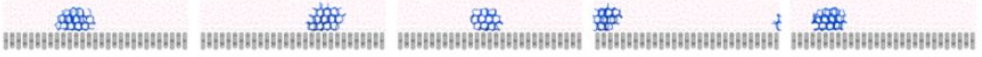

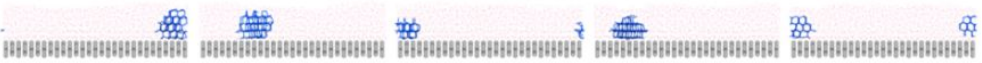

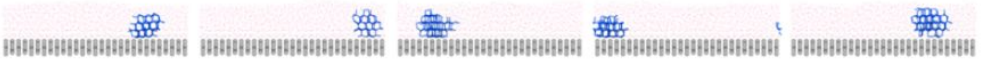

Figure S10. Snapshots of ice nucleation of isothermal simulations in $V_{1} S_{2}$ system. Panel (a), (b) and (c) represent flat surface, step structure and lateral surface respectively. Surfaces are shown with gray balls, liquid water with red dots and ice crystallites with blue sticks. 
(a)

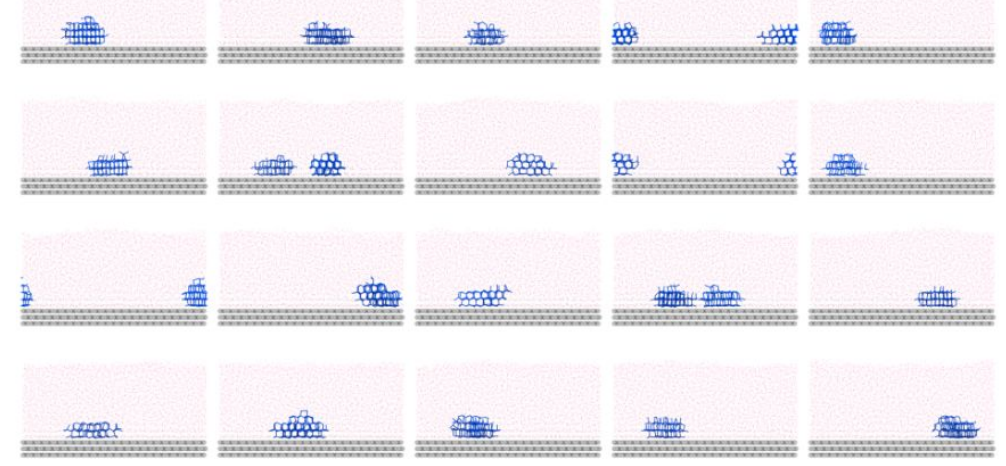

(b)
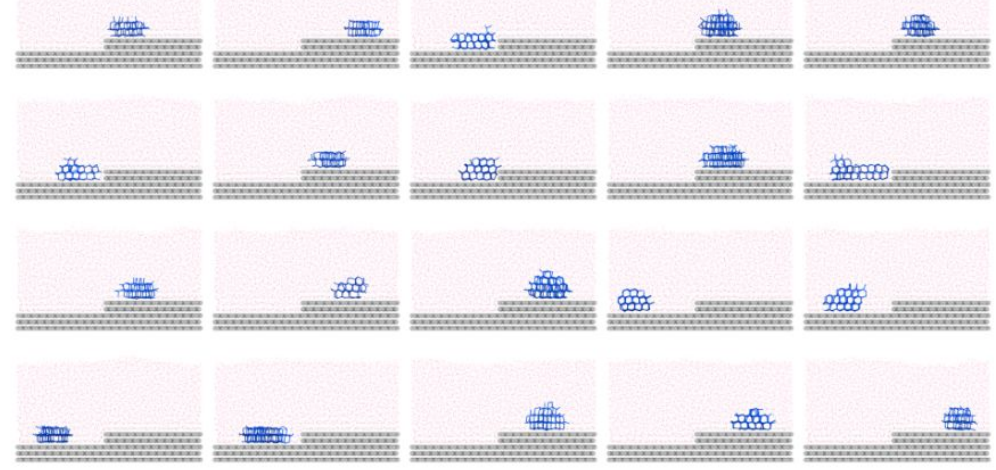

(c)

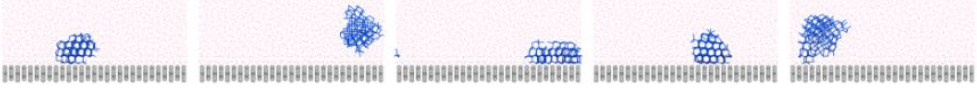

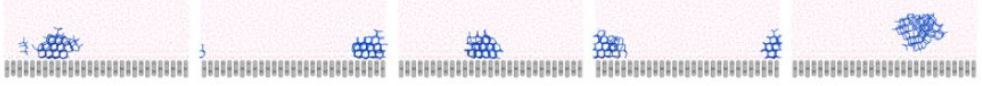

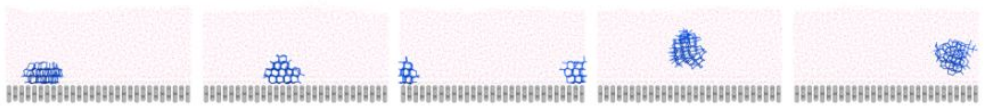

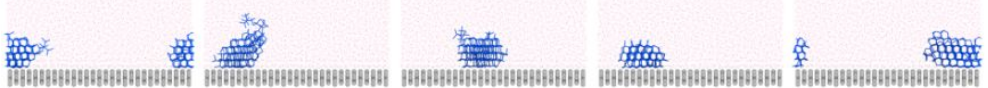

Figure S11. Snapshots of ice nucleation of isothermal simulations in $\mathrm{V}_{2} \mathrm{~S}_{2}$ system. Panel (a), (b) and (c) represent flat surface, step structure and lateral surface respectively. Surfaces are shown with gray balls, liquid water with red dots and ice crystallites with blue sticks. 


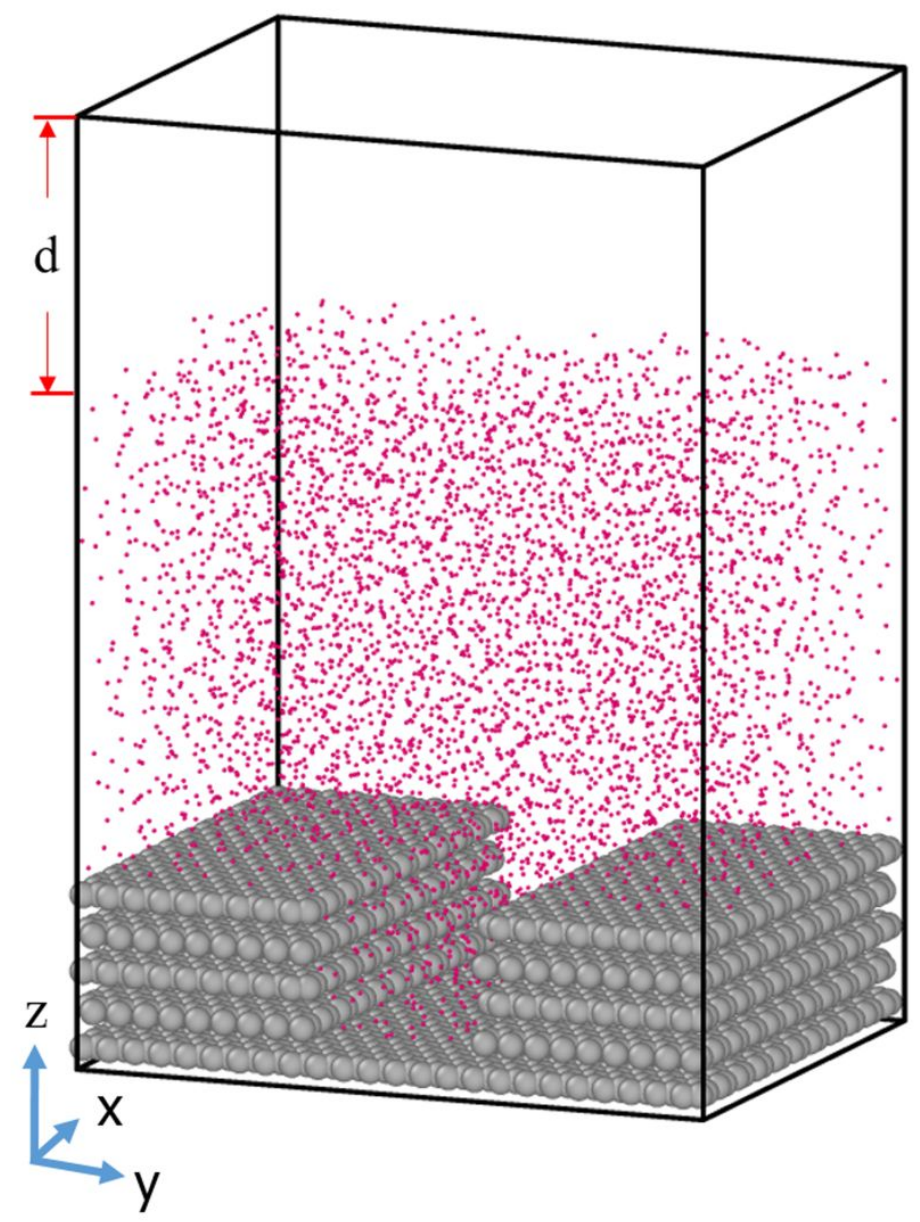

Figure S12. MD simulation system with nanogrooves of graphite. The dimensions of the simulation box are $4.686,5.904$, and $8.5 \mathrm{~nm}$ in the $x, y, z$ directions. Periodic boundary conditions are applied in all directions. The thickness of the vacuum layer above water layer, $d$, is set to $2.3 \mathrm{~nm}$. Surfaces are shown with gray balls, red dots for $\mathrm{mW}$ water molecules. 